
$\$$ Research Square
Preprints are preliminary reports that have not undergone peer review.
They should not be considered conclusive, used to inform clinical practice, or referenced by the media as validated information.

\title{
Chitosan capping enzyme-responsive hollow mesoporous silica nanoplatforms for colon specific drug delivery
}

\section{Defu Cai}

Qiqihar Medical University

\section{Cuiyan Han}

Qiqihar Medical University

Chang Liu

Qiqihar Medical University

\section{Xiaoxing Ma}

Qiqihar Medical University

Jiayi Qian

Qiqihar Medical University

Jianwen Zhou

Qiqihar Medical University

\section{Yue Li}

Heilongjiang hospital of traditional Chinese medicine

\section{Yiming Sun}

Heilongjiang hospital of traditional Chinese medicine

\section{Changting Zhang}

Qiqihar Medical University

Wenquan Zhu ( $\sim$ zhuwenquan1984@126.com )

Qiqihar Medical University

\section{Nano Express}

Keywords: hollow mesoporous silica spheres, colon specific drug delivery, enzyme-responsive, doxorubicin

Posted Date: March 5th, 2020

DOI: https://doi.org/10.21203/rs.3.rs-16212/v1

License: (c) (1) This work is licensed under a Creative Commons Attribution 4.0 International License.

Read Full License 
Version of Record: A version of this preprint was published at Nanoscale Research Letters on June 1st, 2020. See the published version at https://doi.org/10.1186/s11671-020-03351-8. 


\section{Abstract}

In summary, an enzyme-responsive colon specific drug delivery system was developed based on hollow mesoporous silica sphere (HMSS), in which the biodegradable chitosan (CS) was gated on the openings of HMSS through cleavable azo bonds (HMSS-N=N-CS). Doxorubicin (DOX) was encapsulated into the hollow cavity and mesopores of HMSS, and HMSS-N=N-CS/DOX showed a high loading amount of $35.2 \%$. X-ray diffraction (XRD) experiment proved that the DOX loaded in the HMSS-N=N-CS was in a non-crystalline state. In vitro drug release experiments proved that HMSS-N=N-CS/DOX showed an enzyme-responsive drug release property. The grafted CS could increase the biocompatibility and stability of HMSS, and reduce the protein adsorption on the surface of HMSS. The gastrointestinal mucosa irritation and cell cytotoxicity results indicated the good biocompatibility of HMSS and HMSS-N=N-CS. The confocal laser scanning microscope (CLSM) and flow cytometry technique (FCM) results indicated that the cellular uptake of DOX was obviously increased after the HMSS-N=N-CS/DOX was preincubated with colonic enzyme mixture. Cell viability result indicated that HMSS-N=N-CS/DOX incubated with colon enzyme showed an increased cytotoxicity and the IC50 value was three time less than that of HMSS-N=NCS/DOX group. The present work will lay the foundation for subsequent research on mesoporous carriers for oral colon-specific drug delivery.

\section{Introduction}

Recently, stimuli-responsive drug delivery systems (DDSs) have gained extensive attention for efficient loading and selective release of drugs in the targeted diseased tissues [1]. The designed stimuliresponsive systems can be delivered to diseased sites and realize the on-demand drug release to improve therapeutic effect and hinder premature leakage induced side-effects. All manner of internal and external stimuli such as redox potential[2], pH [3], enzyme [4], temperature and light[5] have been used to design stimuli-responsive DDSs. Among these stimuli, enzyme as an internal stimulus has gained wide attention due to the distinct concentrations in different tissues [6].

Over the past two decades, mesoporous silica spheres (MSS) nanomaterials with the mesopores between 2-50 $\mathrm{nm}$ have been established as stimuli-responsive drug carriers [7, 8], since MSS had remarkably large pore volume and high surface area for high drug loading capacity, well-organized pore structure, easily functionalized surface as well as good biocompatibility $[9,10]$. Furthermore, hollow mesoporous silica sphere (HMSS) nanoparticles with the mesoporous shell structure and a hollow cavity are superior to conventional MSS, because the hollow structure can efficiently load more drugs with a higher storage capacity than MSS carriers [11, 12]. And all kinds of stimuli-responsive nanocarriers based on MSS were developed to host drug molecules using various gatekeepers, such as polymers[13], inorganic nanoparticles [14], dendrimers, biomacromolecules [9], macrocyclic compounds peptides [15], and lipids [16]. Although numerous DDS based on MSS with functional capping can realize stimuli-responsive release in response to various external or internal stimuli, few of them have been used in colon-specific targeted drug delivery. 
It is well-known that oral drug delivery is the favorite and simple way of administering drugs. And the colon-specific targeted drug delivery is very fascinating for the treatment of colonic diseases including Crohn's disease, colorectal cancer, ulcerative colitis and so on. However, colon-specific drug delivery might encounter several troubles including less water content compared with the other sites in the gastrointestinal (GI) tract and relatively less surface for oral adsorption in the colonic sites [17-19]. Furthermore, the oral DDSs also meet with strong acidic environment in the stomach, which might accelerate the degradation of loaded drugs in the GI tract, thus losing the ability to realize the colonic targeted delivery [19]. Based on this reason, several $\mathrm{pH}$-dependent DDSs have been designed to realize the $\mathrm{pH}$-triggered drug release near the neutral $\mathrm{pH}$ condition (6-7) of $\mathrm{GI}$ tract, resisting to the highly acidic condition in the stomach region [20-22]. Only a slight difference of acidity between the intestinal ( $\mathrm{pH} 6.8)$ and colonic $(\mathrm{pH} 7.4)$ region is existed, hence such $\mathrm{pH}$-responsive DDSs are difficult to realize the colonspecific release.

Chitosan (CS), a cationic and biodegradable natural present polysaccharide, is constituted by $\beta-(1-4)$ linked glucosamine and $\mathrm{N}$-acetyl-D-glucosamine units[23]. CS has been paid great attention in biological medicine owing to its fascinating properties including biodegradability, nontoxicity and antibacterial activity [24]. Compared with polymers, polyelectrolytes and supramolecules synthesized via complicated processes, CS is relatively cheap and readily available by the exhaustive deacetylation of chitin. In addition, it has been reported that CS can open tight junctions between cells, thus increasing the drug absorption [25]. Therefore, the polymer CS was selected as a capping owing to the good biocompatibility and fitted molecular weight to cover the mesopores of HMSS to block drug release.

In our work, the colon-specific enzyme-responsive DDS based on HMSS (HMSS-N=N-CS) was designed for the first time as displaced in Scheme 1. In this system, the HMSS carriers were prepared via a selective etching strategy. The polymer CS was covered on the surface of HMSS by azo bonds as a gatekeeper to block the openings of HMSS. The azo bonds between HMSS and CS can be cleaved by enzyme in the colon sites, resulting in the separation of CS from the openings of HMSS. DOX was used as the model drugs to be embedded into the cavity of HMSS and the in vitro drug release experiments were conducted to evaluate the enzyme-responsive release in presence of colonic enzyme. The confocal laser scanning microscope (CLSM) and flow cytometry technique (FCM) were used to investigate the cellular uptake by using Caco-2 cells. Finally, the cytotoxicity of HMSS-N=N-CS/DOX was measured towards Caco-2 cells.

\section{Materials/experimental}

\section{Chemistry}

Tetraethoxysilane (TEOS), N-(3-dimethylaminopropyl)-N-ethylcarbodiimide hydrochloride (EDC), chitosan (DAC $\geq 95 \%$ ), cetyltrimethylammonium bromide (CTAB), 3-aminopropyltriethoxysilane (APTES), 3-(4,5Dimethylthiazol-2-yl)-2,5-diphenyl tetrazolium bromide (MTT), azobenzene-3,3'-dicarboxylic acid $\llbracket \mathrm{N}$ hydroxysuccinimide (NHS), DOX and were purchased from Aladdin Chemical Inc.(Shanghai, China). Azobenzene-3,3'-dicarboxylic acid was supplied by Inno-chem Technology Co. Ltd. (Beijing, China). Cell 
culture media DMEM, penicillin-streptomycin and fetal bovine serum (FBS) were supplied by GIBCO, Invitrogen Co. (Carlsbad, USA). All analytical reagents were not further purified before use.

\section{Preparation of HMSS-N=N-CS}

\section{Preparation of HMSS- $\mathrm{NH}_{2}$}

The HMSS nanoparticles were prepared based on the published work using a selective etching method [26]. The solid silica spheres were firstly synthesized by a modified Stober method. Briefly, $6 \mathrm{~mL}$ TEOS was poured into the mixture of $10 \mathrm{~mL}$ deionized water, $74 \mathrm{~mL}$ ethanol, and $3 \mathrm{~mL}$ concentrated $\mathrm{NH}_{3} \cdot \mathrm{H}_{2} \mathrm{O}$. Subsequently, the mixture was stirred for 60 min to obtain colloidal silica suspensions at ambient temperature. The solid spheres were centrifuged, washed and dried for further use. Then, the mesoporous silica shell was covered on solid silica spheres. $300 \mathrm{mg}$ solid silica was dispersed in $50 \mathrm{~mL}$ deionized water by ultra-sonication for $45 \mathrm{~min}$. And the silica suspensions were poured into a mixyure of $60 \mathrm{~mL}$ ethanol, $450 \mathrm{mg}$ CTAB, $90 \mathrm{~mL}$ water and $1.7 \mathrm{~mL} \mathrm{NH} \mathrm{H}_{3} \cdot \mathrm{H}_{2} \mathrm{O}$. After the mixture stirred for $60 \mathrm{~min}$, TEOS $(0.75$ $\mathrm{mL}$ ) was added. Subsequently, the nanoparticles were centrifuged after stirring for $6 \mathrm{~h}$ to collect samples, and then re-dispersed in $40 \mathrm{~mL}$ water. About $1.2 \mathrm{~g} \mathrm{Na}_{2} \mathrm{CO}_{3}$ was added into the water suspension with vigorous stirring. After the mixture was maintained at $55^{\circ} \mathrm{C}$ for $12 \mathrm{~h}$, the products of HMSS nanoparticles were collected and washed with anhydrous ethanol. The post-grafting method with the ratio of HMSS and APTES being at 4:1 (m/v) to prepare HMSS- $\mathrm{NH}_{2}$ at $80{ }^{\circ} \mathrm{C}$ under $\mathrm{N}_{2}$ condition for $8 \mathrm{~h}$. To CTAB was removed by reflux [27].

\section{Preparation of HMSS-N=N-COOH}

The azobenzene-3,3'-dicarboxylic acid ( $50 \mathrm{mg})$ was added in pH 5.8 PBS. Then $(5 \mathrm{mg} / \mathrm{mL})$ EDC and (3 $\mathrm{mg} / \mathrm{mL}$ ) NHS were added to activate azobenzene-3,3'-dicarboxylic acid at $30^{\circ} \mathrm{C}$ for $1 \mathrm{~h}$. And $10 \mathrm{~mL}$ PBS containing $15 \mathrm{mg} / \mathrm{mL}$ HMSS- $\mathrm{NH}_{2}$ was added, and the mixture was stirred for $24 \mathrm{~h}$. And the resultant HMSS-N=N-COOH was separated by centrifugation and washed with ethanol.

\section{Preparation of HMSS-N=N-CS}

$0.15 \mathrm{~g} \mathrm{CS}$ and $0.5 \mathrm{~mL}$ acetic acid were added in $50 \mathrm{~mL}$ water to prepare CS solution. And $100 \mathrm{mg} \mathrm{HMSS}-$ $\mathrm{N}=\mathrm{N}-\mathrm{COOH}$ was dispersed in $25 \mathrm{~mL}$ pH 5.0 PBS and activated by EDC and NHS for $0.5 \mathrm{~h}$. Then CS solution $(10 \mathrm{~mL})$ was poured in the suspension with continuous stirring for $1 \mathrm{~d}$. Finally, the synthesized HMSS-N=N-CS was centrifuged and washed to collect the samples.

\section{Extraction of colonic enzyme mixture from microflora}

The colonic microflora was collected according to a published work [28]. Then the culture was inoculated to get enzyme mixture secreted by colonic microflora at $37^{\circ} \mathrm{C}$. The simulated colonic media containing enzyme mixture was filtered through a $0.22 \mu \mathrm{m}$ filter to remove all the cellular debris from the culture 
fluid. Subsequently, the filtrate was lyophilized to obtain the enzyme mixture in the powder form, which was used in the further study.

\section{Drug loading process and enzyme-responsive release}

$25 \mathrm{mg}$ DOX was dissolved in $5 \mathrm{~mL}$ pH $3.5 \mathrm{HCl}$ solution. And $100 \mathrm{mg} \mathrm{HMSS}-\mathrm{N}=\mathrm{N}-\mathrm{CS}$ was added in the DOX solution, and stirred at ambient temperature for $12 \mathrm{~h}$. Subsequently, $0.2 \mathrm{M} \mathrm{NaOH}$ solution was used to adjust the $\mathrm{pH}$ of mixture to 7.0 , and the suspension was stirred for another $12 \mathrm{~h}$. Then, the DOX-loaded HMSS-N=N-CS (referred to HMSS-N=N-CS/DOX) was centrifuged and washed to remove the adsorbed DOX on the surface of HMSS-N=N-CS. The supernatant was gathered at each step to measure the DOX loading efficiency at $480 \mathrm{~nm}$ by UV-Vis spectrophotometry. The total mass of DOX loaded in HMSS-N=NCS was calculated by subtracting the unloaded DOX after drug loading processes from the initial mass of DOX added.

\section{BSA adsorption}

The BSA adsorption amount was evaluated based on the published works $[29,30]$. BSA was added in $\mathrm{pH}$ 7.4 PBS $(0.5 \mathrm{mg} / \mathrm{mL}) .5 \mathrm{mg} \mathrm{HMSS}$ and $\mathrm{HMSS}-\mathrm{NH}_{2}$ and HMSS-N=N-CS were added into $2.5 \mathrm{~mL}$ PBS (pH 7.4). And the equal volume BSA solution was supplied, and the suspension was placed in a shaker at $100 \mathrm{rpm}$. After $6 \mathrm{~h}$, centrifugation was used to collect the upper solution. At last, the BSA concentration was measured at $595 \mathrm{~nm}$ after being stained with Coomassie brilliant blue solution.

\section{Characterization}

The mesoporous network structure and morphology of the HMSS nanoparticles were evaluated by TEM images (EM-208S, CSIS, USA). The surface area and pore size distribution of nanoparticles were characterized using nitrogen adsorption analysis analyzer (V-Sorb 2800P, Gold APP Instrument Corporation, China). The $\xi$ potentials and particle sizes were characterized on a Nano-z90 Nanosizer (Malvern Instruments Ltd., Worcestershire, UK). TGA analysis was measured on a TGA-50 equipment (Shimadzu, Kyoto, Japan) with a heating rate of $10^{\circ} \mathrm{C} / \mathrm{min}$ under a nitrogen flow. Power XRD was performed on a Siemens D5005 X-ray diffractometer (Karlsruhe, Germany) with Cu-Ka radiation ( $\lambda=$ $1.5418 \AA$ ).

\section{Cell culture and cell uptake experiment}

Caco-2 cells were cultured in a medium supplemented with $10 \%$ FBS, $1 \%$ nonessential amino-acid, $1 \%$ (v/v) pyruvic acid sodium and $1 \%$ streptomycin. NIH-3T3 cells were cultured in DMEM with $1 \%$ streptomycin and $10 \%$ FBS. The Caco- 2 cells uptake of the nanocarriers was characterized using FCM and CLSM. Caco-2 cells were seeded into 24-well plates. After culturing for overnight, free DOX, HMSS$\mathrm{N}=\mathrm{N}-\mathrm{CS} / \mathrm{DOX}$ and HMSS-N=N-CS/DOX pre-incubated with colonic enzyme nanoparticles (equal to the concentration of $5 \mu \mathrm{g} / \mathrm{mL}$ DOX) were added to corresponding wells. After continued incubation for $2 \mathrm{~h}$, the cell medium was removed and washed thoroughly with PBS. Then, the cells were fixed by $4 \%$ 
formaldehyde and stained by Hoechst 33258 for CLSM observation. FCM was used to obtain a quantitative evaluation of cellular uptake. Caco-2 cells were seeded in 6-well plates and further incubated for $24 \mathrm{~h}$. After washing with PBS, the Caco-2 cells were incubated with free DOX, HMSS-N=N-CS/DOX and HMSS-N=N-CS/DOX pre-incubated with colonic enzyme nanoparticles (equal to the concentration of 5 $\mu \mathrm{g} / \mathrm{mL}$ DOX) in serum-free DMEM for $2 \mathrm{~h}$. Then, the Caco-2 cells were rinsed with cold PBS, trypsinized and re-suspended in $0.5 \mathrm{~mL}$ PBS. The DOX fluorescence in cells was measured using a FACSCanto flow cytometer (Becton, Dickinson, USA).

\section{In vitro cellular proliferation assay}

The cytotoxicity of HMSS and HMSS-N=N-CS blank carriers towards NIH-3T3 and Caco-2 cells were testified by MTT assay [31, 32]. Briefly, Caco-2 cells and NIH-3T3 cells were separately seeded in 96-well plates and further incubated for overnight. The old cell medium was substituted by a serum-free medium containing different concentrations of nanoparticles. After incubation for $2 \mathrm{~d}, 50 \mu \mathrm{L}$ of MTT solution (2 $\mathrm{mg} \mathrm{mL}^{-1}$ ) was added and incubated for $4 \mathrm{~h}$ to measure the living cells. Then, MTT solution was removed and $150 \mu \mathrm{L}$ DMSO was added to dissolve formazan. Subsequently, the absorbance was measured on a microplate reader (Tecan, Männedorf, Switzerland) at $570 \mathrm{~nm}$. The cytotoxicity of free DOX, HMSS-N=NCS/DOX and HMSS-N=N-CS/DOX pre-incubated with enzyme mixtures extracted from colonic microflora was measured using Caco-2 cells with the corresponding DOX concentrations of $(0.1,1,5,10$ and 20 $\mu \mathrm{g} / \mathrm{mL}$ ). The incubation time was $48 \mathrm{~h}$, and the other experiment processes were the same as above described.

\section{Toxicity studies}

The gastrointestinal mucosa irritation tests are vital for the evaluation of oral drug delivery in vivo biosafety. Male Sprague-Dawley rats $(180 \pm 10 \mathrm{~g})$ were randomly divided into three groups (three rats for each group). Rats were administrated saline, HMSS and HMSS-N=N-CS nanoparticles with a dose of 100 $\mathrm{mg} / \mathrm{kg}$ for each day. After $7 \mathrm{~d}$, all the rats were sacrificed, and the tissues were collected and examined by histopathological examination (H\&E). To evaluate the biosecurity of HMSS and HMSS-N=N-CS nanoparticles, the body weights of BALB/c mice (18-20 g) were recorded after oral administration at a dose of $100 \mathrm{mg} / \mathrm{kg}$ for every other day.

\section{Results And Discussion}

\section{Preparation and characterization of HMSS-N=N-CS}

The HMSS was prepared based on previous works with minor changes[26]. Firstly, the solid $\mathrm{SiO}_{2}$ nanospheres were prepared, and the mesoporous shell was coated on the surface of the solid silica nanospheres containing CTAB template. Then, the $\mathrm{Na}_{2} \mathrm{CO}_{3}$ was used to selectively etch the solid $\mathrm{SiO}_{2}$ nano-spheres, while the mesoporous shell was protected by template CTAB. The prepared process of HMSS-N=N-CS with CS as a "gatekeeper" by the azo linkage is described in Fig. 1A. First, the surface of 
HMSS was modified with APTES as an alkyl coupling reagent to become amino-functionalized HMSS $\left(\mathrm{HMSS}-\mathrm{NH}_{2}\right)$ by the post-modified method. Subsequently, the $\mathrm{HMSS}-\mathrm{N}=\mathrm{N}-\mathrm{COOH}$ was prepared by an amidation reaction between the amino groups of HMSS- $\mathrm{NH}_{2}$ and the carboxyl groups of azobenzene-3,3'dicarboxylic acid. Then, CS was covalently modified onto the surface of HMSS nanoparticles by an amidation reaction between the carboxyl groups of $\mathrm{HMSS}-\mathrm{N}=\mathrm{N}-\mathrm{COOH}$ and the amino groups in $\mathrm{CS}$.

As displayed in the transmission electron microscopy (TEM) image in Fig. 1A, the average diameter of HMSS was $250 \mathrm{~nm}$ with a uniform hollow structure and highly ordered mesoporous shell. The average mesoporous shell thickness was ca. $90 \mathrm{~nm}$. Compared to the smooth surface of HMSS, the surface of grafted polymer HMSS-N=N-CS (Fig. 1B) was rough, indicating that the CS was covered on the HMSS carrier.

The surface areas and the pore distributions of mesoporous materials played a crucial role in loading and delivering host molecules for controlled release. The pore size distribution curves and isotherms were measured by $\mathrm{N}_{2}$ adsorption and desorption analysis (Fig. 2). The detailed parameters of Brunauer-Emmett-Teller (BET) surface area $\left(\mathrm{S}_{\mathrm{BET}}\right)$, total pore volume $\left(\mathrm{V}_{\mathrm{P}}\right)$ and pore size distribution $\left(\mathrm{D}_{\mathrm{P}}\right)$ are displaced in Table 1. The $\mathrm{S}_{\mathrm{BET}}$ and $V_{P}$ of pure HMSS were $810.7 \mathrm{~m}^{2} / \mathrm{g}$ and $0.969 \mathrm{~cm}^{3} / \mathrm{g}$, respectively, and the $\mathrm{D}_{\mathrm{P}}$ was about $3.8 \mathrm{~nm}$. The $\mathrm{D}_{\mathrm{P}}$ of HMSS- $\mathrm{NH}_{2}$ was almost the same as that of HMSS after amination, indicating that the mesopores were not blocked after amino-functionalization. While $\mathrm{S}_{\mathrm{BET}}$ and $\mathrm{V}_{\mathrm{P}}$ of HMSS-N=N-CS were markedly decreased after modification of azo compound and CS coating, indicating that CS had coated on the surface of the HMSS.

Table 1. The $\mathrm{N}_{2}$ adsorption and desorption parameters of different functionalized HMSS nanoparticles

\begin{tabular}{cccc}
\hline Sample & $\mathrm{S}_{\mathrm{BET}}\left(\mathrm{m}^{2} / \mathrm{g}\right)$ & $\mathrm{V}_{\mathbf{P}}\left(\mathrm{cm}^{3} / \mathrm{g}\right)$ & $\mathrm{D}_{\mathrm{p}}(\mathrm{nm})$ \\
\hline HMSS & 810.7 & 0.969 & $3.8 ; 2.2$ \\
HMSS-NH & 688.1 & 0.822 & $3.8 ; 2.2$ \\
HMSS-N=N-CS & 437.6 & 0.406 & $2.2 ; 3.8$ \\
\hline
\end{tabular}

The successful grafting of HMSS-N=N-CS was verified by various methods. The $\xi$ potential of HMSS$\mathrm{NH}_{2}$ underwent great change after functionalization, varying from $-27.9 \mathrm{mV}$ to $+31.4 \mathrm{mV}$ as shown in Fig. $3 \mathrm{~A}$, which was ascribed to the amine groups on the surface of HMSS. After $\mathrm{HMSS}-\mathrm{NH}_{2}$ reacted with azobenzene-3,3'-dicarboxylic acid, the $\xi$ potential of HMSS-N=N-COOH was further decreased to $-2.0 \mathrm{mV}$ 
because of the carboxyl groups on the surface of HMSS. After CS polymer was further grafted on the surface of HMSS, the $\xi$ potential of HMSS-N=N-CS was reversed to $+32.4 \mathrm{mV}$. The result was ascribed to that amino-abundant positively charged CS coated on the surface of HMSS.

The thermogravimetric analysis (TGA) curves of HMSS, $\mathrm{HMSS}_{-} \mathrm{NH}_{2}, \mathrm{HMSS}-\mathrm{N}=\mathrm{N}-\mathrm{COOH}$ and HMSS-N=N-CS are shown in Fig. 3B. Compared with HMSS-N=N-COOH, an additional weight loss of HMSS-N=N-CS compared with HMSS-N=N-COOH was about $19 \%$, which was due to the removal of CS chains. The grafting of azo bonds on the surface of HMSS was also confirmed by the color change during the preparation of HMSS-N=N$\mathrm{COOH}$ as shown in the inset in Fig. 3B. The reactant $\mathrm{HMSS}-\mathrm{NH}_{2}$ is white, while the product HMSS-N=N-COOH is yellowish-brown after the azobenzene-3,3'-dicarboxylic acid reacted with the amino of HMSS. The hydrodynamic diameters $\left(\mathrm{D}_{\mathrm{H}}\right)$ and polydispersity index (PDI) of HMSS, HMSS-NH $\mathrm{NH}_{2}$ and HMSS-N=N-CS were measured in distilled water as shown in Fig. 3C and 3D. The HMSS had a diameter of $287 \mathrm{~nm}$ with a PDI of 0.284 . After the functionalization of amine groups on the surface of HMSS, the $\mathrm{D}_{\mathrm{H}}$ of $\mathrm{HMSS}-\mathrm{NH}_{2}$ increased to $324 \mathrm{~nm}$. And the diameter of HMSS-N=N-CS was $342 \mathrm{~nm}$, which was larger than that of HMSS-NH $\mathrm{N}_{2}$ due to the grafted CS chains. And the PDI of HMSS-N=N-CS (0.177) was smaller than that of HMSS and HMSS- $\mathrm{NH}_{2}$, indicating that the average particle sizes were more even after the grafting of CS. Compared with the diameter obtained from TEM, the diameters of HMSS and HMSS-N=N-CS measured by DLS were larger. Because the $\mathrm{D}_{\mathrm{H}}$ of the nanoparticles was measured in water environment with a hydration layer, while the size of the nanoparticles provided by TEM was obtained in a dried state. All the results proved the successful preparation of HMSS-N=N-CS.

\section{Drug existing state and loading efficiency}

DOX was chosen to investigate the loading and release behaviors of HMSS-N=N-CS. When the $\mathrm{pH}$ value of HMSS-N=N-CS nanoparticles suspension was adjusted to $\mathrm{pH} 3.5$, CS biomacromolecule polymer became positively charged (the pK of CS is 6.3), owing to the protonated amino groups in acidic environment [23]. The CS polymer became positively charged and swelled, leading to the opening of mesopores of HMSS attributed to the 
repulsive interaction between CS. Thus, DOX got the access to diffusion into the mesopores of HMSS-N=N-CS. However, after the drug-loaded mixture was adjusted to 7.4, the CS chains became deprotonated and collapsed to hinder the premature release of the DOX.

The loading efficiency of HMSS-N=N-CS/DOX was 35.2\%, which was much larger than the other DOX loaded mesoporous silica delivery systems [16, 33]. The high DOX loading efficiency of HMSS nanocarriers was attributed to hollow cavity, large surface area and mesoporous network, which could be used as a drug reservoir. The physical existing state of DOX in HMSS-N=N-CS/DOX was evaluated by Power X-ray diffraction (XRD). As shown in the XRD profiles (Fig. 4), raw DOX exhibited characteristic and intense drug crystalline diffraction peaks. The physical mixture (MP) of HMSS-N=N-CS and DOX also showed obvious crystalline diffraction peaks. However, no distinct crystalline peaks were existed in the HMSS-N=N-CS/DOX, which proved that the physical state of DOX in HMSS-N=NCS/DOX was in a non-crystalline state because of the restricted effect of the mesoporous structure of HMSS.

\section{In vitro enzyme-responsive release in simulated colonic environment}

To investigate the enzyme-responsive release of HMSS-N=N-CS, DOX-loaded HMSS$\mathrm{N}=\mathrm{N}-\mathrm{CS} / \mathrm{DOX}$ nanoparticles were added in $\mathrm{pH}$ 7.4 PBS with different concentrations of colonic enzyme mixture. As displayed in Fig. 5A, HMSS-N=N-CS/DOX exhibited the slow release of DOX at PBS pH 7.4, and the cumulative release percentage was only about $10 \%$ within $24 \mathrm{~h}$, indicating a good capping capability of the covered CS polymers and azo bonds. Expectedly, in case of dilute enzyme at PBS pH 7.4, the cumulative release of DOX was improved to more than $20 \%$ within the same period. Additionally, the release amount of DOX was dramatically increased to nearly $40 \%$ in presence of concentrated enzyme. The relatively low drug release percentage was owing to the electrostatic interaction between the negatively charged HMSS and the positively charged DOX [34]. The above results proved that the release of DOX from HMSS-N=N-CS/DOX was markedly accelerated by extracted enzymes from microflora in colonic regions. And the enzyme-responsive release mechanism could be attributed to the degradation of azo bonds in HMSS-N=N-CS by 
enzyme, causing the detachment of CS from the surface of HMSS and the fast release from HMSS. Since azo bonds have been reported to be cleaved by enzymes secreted by colonic microflora [35, 36].

Additionally, to further evaluate the enzyme-responsive release from HMSS-N=N-CS/DOX in the mimetic GIT environment, the HMSS-N=N-CS/DOX nanoplatforms were initially dispersed in SGF for $2 \mathrm{~h}$, and then further dispersed in SIF for $6 \mathrm{~h}$, and finally the carriers were added in pH7.4 PBS containing $1 \mathrm{mg} / \mathrm{mL}$ extracted enzyme. As shown in Fig. 5B, in simulated gastric juice, the release of DOX showed a relatively fast release rate and the cumulative amount was up to 15\% within $2 \mathrm{~h}$. The relatively fast release was due to the weaker interaction between HMSS-N=N-CS and DOX in the acid conditions[1]. Then, the release rate of DOX was slowed down in SIF for 2-8 h. However, after the HMSS-N=NCS/DOX was incubated with extracted enzymes in pH7.4 PBS, the release of DOX was continued to increase markedly, and the cumulative release amount was more than $50 \%$ within $24 \mathrm{~h}$. The incomplete DOX release form HMSS-N=N-CS/DOX was due to the strong interaction between the positively charged DOX and negatively charged HMSS.

\section{The protein adsorption and stability of HMSS-N=N-CS}

For oral administration, the surface properties of nanocarriers will unavoidably affect the drug release behaviors and bioadsorption [37]. The protein adsorption assay on the surface was used to evaluate the effect of grafted CS on the surface of HMSS. As displayed in Fig. 6A, bare HMSS nanocarriers had a dramatic BSA adsorbance up to $16.5 \%$ attributed to the large surface area and hollow cavity of HMSS, strong adsorption ability and nonspecific interactions between silanol groups of HMSS and BSA [29, 30]. In addition, HMSS- $\mathrm{NH}_{2}$ similarly had a relatively high adsorbed BSA amount of $10.2 \%$. Nevertheless, the adsorbed BSA percentage on the surface of HMSS-N=N-CS markedly decreased to $2.5 \%$ after the covering of polymer CS, thus decreasing dramatically the effect on in vivo behavior of HMSS-N=N-CS. To further observe the stability of HMSS-N=N-CS and HMSS samples, $20 \mathrm{mg}$ of HMSS-N=N-CS and HMSS were added in the pH 7.4 PBS and deionized water. As displayed in Fig. 6B, although HMSS-N=N-CS and HMSS were relatively stable 
in water, HMSS quickly flocculated in pH 7.4 PBS. By contrast, the dispersity of HMSS$\mathrm{N}=\mathrm{N}$-CS was obviously enhanced after the grafting polymer CS on the surface of HMSS. Additionally, HMSS-N=N-CS carriers can keep stable for more than $12 \mathrm{~h}$ without precipitation at $\mathrm{pH}$ 7.4 PBS. These results proved that the covering of hydrophilic CS polymer could improve the dispersity and decrease proteins adsorption on the surface of HMSS-N=N-CS.

\section{Cellular uptake}

Caco-2 cells as the human epithelial colorectal adenocarcinoma cells are widely used as the model cells in oral drug delivery. As shown in Fig. 7A, Caco-2 cells incubated with free DOX showed a relatively strong fluorescence signal resulted from DOX, because positively charged DOX could enter the cell and be located in the nucleus. Compared with free DOX group, HMSS-N=N-CS/DOX group showed a weaker fluorescence signal intensity due to the incomplete drug release from HMSS-N=N-CS/DOX. However, after HMSS-N=NCS/DOX was pre-incubated with colonic enzyme mixture for $1 \mathrm{~h}$, the HMSS-N=N-CS/DOX + enzyme group showed the markedly increased fluorescence signal. This was attributed to that the azo bonds were cleaved by the enzyme mixture which lead to the fall off the CS from the surface of HMSS, thus accelerating significantly the DOX release from HMSS$\mathrm{N}=\mathrm{N}-\mathrm{CS} / \mathrm{DOX}$. To quantitatively evaluate the cellular uptake differences for HMSS-N=NCS/DOX and HMSS-N=N-CS/DOX, the FCM was further used. As shown in Fig. 7B, the mean fluorescence intensity (MFI) for HMSS-N=N-CS/DOX group was 124.7, which was weaker compared with the free DOX group with a p value less than 0.001. Excitingly, after HMSS-N=N-CS/DOX was pre-incubated with colonic enzyme mixture for $1 \mathrm{~h}$, MFI for HMSS-N=N-CS/DOX + enzyme group markedly increased to 357 and even exceeded the free DOX group owing to the accelerated drug release from HMSS-N=N-CS/DOX after the breakage of azo bonds. All these results indicated that the azo bonds in HMSS-N=NCS/DOX could be cleaved in the presence of colonic enzyme, which will lead to the shedding of CS from the surface of HMSS and accelerate the DOX release from HMSS.

\section{In vitro cell viability evaluation}


To prove the enzyme-responsive release effect of HMSS-N=N-CS/DOX in simulated colonic condition, the in vitro cell viability assay was carried out by using Caco-2 cell. And the classic anticancer drug DOX was used in the cell viability assay. Prior to this assay, the different concentrations of blank HMSS and HMSS-N=N-CS were employed to ascertain the biocompatibility of nanoplatform towards Caco-2 cells and normal NIH-3T3 (mouse embryo fibroblast) cells with various concentrations from 10 to $250 \mu \mathrm{g} / \mathrm{mL}$ by MTT assay [38, 39]. As displayed in Fig. 8 A and B, HMSS and HMSS-N=N-CS showed negligible cytotoxicity after incubation with Caco-2 cells or NIH-3T3 cells with different concentrations, and the viability of Caco-2 cells were $87.9 \%$ and $88.3 \%$ at a high concentration of $100 \mu \mathrm{g} / \mathrm{mL}$, which is high sufficiently for clinical applications due to the high drug loading of HMSS. In addition, the NIH-3T3 cells incubated with HMSS and HMSS-N=N-CS for $48 \mathrm{~h}$ had the high cell viability above $80 \%$ at a relatively high concentration of $100 \mu \mathrm{g} / \mathrm{mL}$. These results indicated that HMSS and HMSS-N=N-CS are cytocompatibility and could be employed for oral delivery.

Effect of DOX loaded HMSS-N=N-CS/DOX on the viability of Caco-2 cells was displayed in Fig. 8C. Free DOX showed a strong and concentration-dependent cytotoxicity towards $4 \mathrm{~T} 1$ cells, which was attributed to that positively charged free DOX could pass through cell membranes easily. The $\mathrm{IC}_{50}$ value

for free DOX group was $10.18 \mu \mathrm{g} \mathrm{mL}^{-1}$ by using SPSS Statistics software. Compared with free DOX at the same DOX concentration, HMSS-N=N-CS/DOX exhibited a higher cell viability than free DOX because of the relatively strong electrostatic interactions between HMSS and DOX carriers and incompletely release of DOX from HMSS-N=N-CS. And $\mathrm{IC}_{50}$ value for HMSS-N=N-CS/DOX group was $32.22 \mu \mathrm{g} \mathrm{mL}^{-1}$, much higher than that for free DOX. However, HMSS-N=N-CS/DOX preincubated with colon enzyme group showed a concentration-dependent cytotoxicity and the $\mathrm{IC}_{50}$ value was calculated to be $9.41 \mu \mathrm{g} \mathrm{mL}^{-1}$. This reason could be ascribed to that the colon enzyme could degrade the azo bonds in HMSS-N=N-CS, which would lead to the detachment of grafted CS from the surface of HMSS, causing the fast release of DOX from HMSS.

\section{Toxicity studies}

The safety of HMSS-N=N-CS is a vital factor to be considered before its clinical applications in future. $\mathrm{H} \& \mathrm{E}$ of gastrointestinal mucosa irritation is essential to evaluate the 
in vivo biosafety of the delivery system for oral administration (Fig. 9A). Compared to saline group, both HMSS and HMSS-N=N-CS groups exhibited no markedly histopathological changes or hyperemia after oral administration for a week with the administration dose of $100 \mathrm{mg} / \mathrm{kg}$. No death or unusual behaviors of rats were observed during the experiment process. The decrease in body weight is widely regarded as an important and simple index for in vivo systemic toxicity [40]. As shown in Fig. 9B, the body weights of mice for HMSS and HMSS-N=N-CS groups increased slightly and were similar as the saline group. The above results indicated that HMSS and HMSS-N=N-CS showed the good biocompatibility as drug carriers for oral administration.

\section{Conclusions}

In summary, an enzyme-responsive colon specific drug delivery has been developed, in which the biodegradable CS was gated on the openings of HMSS through cleavable azo bonds. DOX was encapsulated into the hollow cavity and mesopores of HMSS, and HMSS-N=N-CS/DOX showed a high loading amount of $35.2 \%$. XRD experiment proved that the drug was in a non-crystalline state in the HMSS-N=N-CS/DOX. In vitro release results proved that HMSS-N=N-CS/DOX showed an enzymeresponsive drug release behavior. Stability and BSA adsorption results illustrated that the grated CS could increase the biocompatibility and stability of HMSS. The cell cytotoxicity and gastrointestinal mucosa irritation results indicated the good biocompatibility of HMSS and HMSS-N=N-CS. The CLSM uptake and FCM results indicated that the cellular uptake of DOX was obviously increased after the HMSS-N=N$\mathrm{CS} / \mathrm{DOX}$ was incubated with colonic enzyme mixture. Cell viability result indicated that HMSS-N=NCS/DOX incubated with colon enzyme showed an increased cytotoxicity and the $\mathrm{IC}_{50}$ value was three time less than that of HMSS-N=N-CS/DOX group. The present work will lay the foundation for subsequent research on inorganic mesoporous carriers for oral colon-specific drug delivery.

\section{Declarations}

\section{Disclosure statement}

The authors report no conflicts of interest

Acknowledgments: This work was supported by University Nursing Program for Young Scholars with Creative Talents in Heilongjiang Province (No. UNPYSCT-2018029).

\section{Authors' Contributions}

ZW, CD, HC, and LC participated in the design of the study. ZW, CD, HC, LC, MX, QJ, ZJ, LY, SY, and ZC performed the experiments and materials characterization. ZW and CD drafted the manuscript. ZW 
provided financial supports. All authors read and approved the final manuscript.

\section{Availability of data and materials}

All data are fully available without restriction

\section{References}

[1] Jiao J, Li X, Zhang S, Liu J, Di D, Zhang Y, et al. (2016) Redox and pH dual-responsive PEG and chitosan-conjugated hollow mesoporous silica for controlled drug release. Mater Sci Eng C 67:26-33.

[2] Zhang J, Yuan ZF, Wang Y, Chen WH, Luo GF, Cheng SX, et al. (2013) Multifunctional envelope-type mesoporous silica nanoparticles for tumor-triggered targeting drug delivery. J Am Chem Soc 135: 50685073.

[3] Jiao J, Li X, Zhang S, Liu J, Di D, Zhang Y, et al. (2016) Redox and pH dual-responsive PEG and chitosan-conjugated hollow mesoporous silica for controlled drug release. Mater Sci Eng C 67:26-33.

[4] Zhao Q, Jia L, Zhu W, Sun C, Di D, Ying Z, et al. (2015) Dual-stimuli responsive hyaluronic acidconjugated mesoporous silica for targeted delivery to CD44-overexpressing cancer cells. Acta Biomaterialia 23:147-56.

[5] Qin Y, Chen J, Bi Y, Xu X, Zhou H, Gao J, et al. (2015) Near-infrared light remote-controlled intracellular anti-cancer drug delivery using thermo/pH sensitive nanovehicle. Acta Biomaterialia 17:201-209.

[6] Cheng R, Feng F, Meng F, Deng C, Feijen J, Zhong Z. (2011) Glutathione-responsive nano-vehicles as a promising platform for targeted intracellular drug and gene delivery. J Control Release 152:2-12.

[7] Tang F, Li L, Chen D. (2012) Mesoporous silica nanoparticles: synthesis, biocompatibility and drug delivery. Adv Mater 24:1504-34.

[8] Wang Y, Zhao Q, Han N, Bai L, Li J, Liu J, et al. (2015) Mesoporous silica nanoparticles in drug delivery and biomedical applications. Nanomed Nanotechnol 11:313-27.

[9] Zhao Q, Wang C, Liu Y, Wang J, Gao Y, Zhang X, et al. (2014) PEGylated mesoporous silica as a redoxresponsive drug delivery system for loading thiol-containing drugs. Int J Pharm 477:613-622.

[10] Lei W, Sun C, Jiang T, Gao Y, Yang Y, Zhao Q, et al. (2019) Polydopamine-coated mesoporous silica nanoparticles for multi-responsive drug delivery and combined chemo-photothermal therapy. Mater Sci Eng C;105:110103.

[11] Hadipour Moghaddam SP, Yazdimamaghani M, Ghandehari H. (2018) Glutathione-sensitive hollow mesoporous silica nanoparticles for controlled drug delivery. J Control Release 282:62-75. 
[12] Wu Y, Lu J, Mao Y, Jiang T, Zhao Q, Wang S. (2020) Composite phospholipid-coated hollow mesoporous silica nanoplatform with multi-stimuli responsiveness for combined chemo-photothermal therapy. J Mater Sci 1-17.

[13] Radu DR, Lai C-Y, Jeftinija K, Rowe EW, Jeftinija S, Lin VS-Y. (2004) A polyamidoamine dendrimercapped mesoporous silica nanosphere-based gene transfection reagent. J Am Chem Soc 126:1321613227.

[14] Yang Y, Lin Y, Di D, Zhang X, Wang D, Zhao Q, et al. (2017) Gold nanoparticle-gated mesoporous silica as redox-triggered drug delivery for chemo-photothermal synergistic therapy. J Colloid Interface Sci 508: 323-331.

[15] Sun YL, Yang BJ, Zhang SXA, Yang YW. (2012) Cucurbit [7] uril Pseudorotaxane-Based Photoresponsive Supramolecular Nanovalve. Chem-A Eur J 18:9212-9216.

[16] Han N, Zhao Q, Wan L, Wang Y, Gao Y, Wang P, et al. (2015) Hybrid lipid-capped mesoporous silica for stimuli-responsive drug release and overcoming multidrug resistance. ACS Appl Mater Interfaces 7:33423351.

[17] Tannergren C, Bergendal A, Lennernäs H, Abrahamsson B. (2009) Toward an increased understanding of the barriers to colonic drug absorption in humans: implications for early controlled release candidate assessment. Mol Pharm 6:60-73.

[18] Friend DR. (1991) Colon-specific drug delivery. Advanced drug delivery reviews;7:149-199.

[19] Park JH, Saravanakumar G, Kim K, Kwon IC. (2010) Targeted delivery of low molecular drugs using chitosan and its derivatives. Adv Drug Deliv Rev 62:28-41.

[20] Prajakta D, Ratnesh J, Chandan K, Suresh S, Grace S, Meera V, et al. (2009) Curcumin loaded pHsensitive nanoparticles for the treatment of colon cancer. J Biomed Nanotech 5:445-455.

[21] Cerchiara T, Abruzzo A, Di Cagno M, Bigucci F, Bauer-Brandl A, Parolin C, et al. (2015) Chitosan based micro-and nanoparticles for colon-targeted delivery of vancomycin prepared by alternative processing methods. Eur J Pharm Biopharm 92:112-119.

[22] Chaturvedi K, Kulkarni AR, Aminabhavi TM. (2011) Blend microspheres of poly (3-hydroxybutyrate) and cellulose acetate phthalate for colon delivery of 5-fluorouracil. Industrial Eng Chem Res 50:1041410423.

[23] Bhattarai N, Gunn J, Zhang M. (2010) Chitosan-based hydrogels for controlled, localized drug delivery. Adv Drug Deliv Rev 62:83-99.

[24] Wu J, Sailor MJ. (2009) Chitosan Hydrogel-Capped Porous SiO2 as a pH Responsive Nano-Valve for Triggered Release of Insulin. Adv Funct Mater 19:733-741. 
[25] Sonaje K, Chuang EY, Lin KJ, Yen TC, Su FY, Tseng MT, et al. (2012) Opening of epithelial tight junctions and enhancement of paracellular permeation by chitosan: microscopic, ultrastructural, and computed-tomographic observations. Mol Pharm 9:1271-1279.

[26] Fang X, Chen C, Liu Z, Liu P, Zheng N. (2011) A cationic surfactant assisted selective etching strategy to hollow mesoporous silica spheres. Nanoscale 3:1632-1639.

[27] Zhao QF, Liu J, Zhu WQ, Sun CS, Di DH, Zhang Y, et al. (2015) Dual-stimuli responsive hyaluronic acid-conjugated mesoporous silica for targeted delivery to CD44-overexpressing cancer cells. Acta Biomater 23:147-156.

[28] Agarwal T, Narayana SN, Pal K, Pramanik K, Giri S, Banerjee I. (2015) Calcium alginate-carboxymethyl cellulose beads for colon-targeted drug delivery. Int J Biological Macromol 75:409-417.

[29] He Q, Zhang J, Shi J, Zhu Z, Zhang L, Bu W, et al. (2010) The effect of PEGylation of mesoporous silica nanoparticles on nonspecific binding of serum proteins and cellular responses. Biomaterials 31:1085-1092.

[30] Gu J, Su S, Zhu M, Li Y, Zhao W, Duan Y, et al. (2012) Targeted doxorubicin delivery to liver cancer cells by PEGylated mesoporous silica nanoparticles with a pH-dependent release profile. Micropor Mesopor Mater 161:160-167.

[31] Wan L, Wang X, Zhu W, Zhang C, Song A, Sun C, et al. (2015) Folate-polyethyleneimine functionalized mesoporous carbon nanoparticles for enhancing oral bioavailability of paclitaxel. Int J Pharm 484:207217.

[32] Zhang Y, Zhang H, Che E, Zhang L, Han J, Yang Y, et al. (2015) Development of novel mesoporous nanomatrix-supported lipid bilayers for oral sustained delivery of the water-insoluble drug, lovastatin. Colloids Surf B 128:77-85.

[33] Zhao Q, Liu J, Zhu W, Sun C, Di D, Zhang Y, et al. (2015) Dual-stimuli responsive hyaluronic acidconjugated mesoporous silica for targeted delivery to CD44-overexpressing cancer cells. Acta Biomaterialia 23:147-156.

[34] Tang H, Guo J, Sun Y, Chang B, Ren Q, Yang W. (2011) Facile synthesis of pH sensitive polymercoated mesoporous silica nanoparticles and their application in drug delivery. Int J Pharm 421:388-396.

[35] Popat A, Ross BP, Liu J, Jambhrunkar S, Kleitz F, Qiao SZ. (2012) Enzyme-responsive controlled release of covalently bound prodrug from functional mesoporous silica nanospheres. Angew Chem Int Edit 51:12486-9.

[36] Rao J, Khan A. (2013) Enzyme sensitive synthetic polymer micelles based on the azobenzene motif. J Am Chem Soc 135:14056-9. 
[37] Popat A, Hartono SB, Stahr F, Jian L, Shi ZQ, Gao QL. (2011) Mesoporous silica nanoparticles for bioadsorption, enzyme immobilisation, and delivery carriers. Nanoscale 3:2801-2818.

[38] Chai GH, Xu Y, Chen SQ, Cheng B, Hu FQ, You J, et al. (2016) Transport Mechanisms of Solid Lipid Nanoparticles across Caco-2 Cell Monolayers and their Related Cytotoxicology. ACS Applied Mater Interf 8:5929-5940.

[39] Peng Y, Yadava P, Heikkinen AT, Parrott N, Railkar A. (2014) Applications of a 7-day Caco-2 cell model in drug discovery and development. Eur J Pharm Sci 56:120-130.

[40] Er-Yuan C, Kun-Ju L, Fang-Yi S, Fwu-Long M, Barnali M, Chiung-Tong C, et al. (2013) Noninvasive imaging oral absorption of insulin delivered by nanoparticles and its stimulated glucose utilization in controlling postprandial hyperglycemia during OGTT in diabetic rats. J Control Release 172:513-522.

\section{Figures}
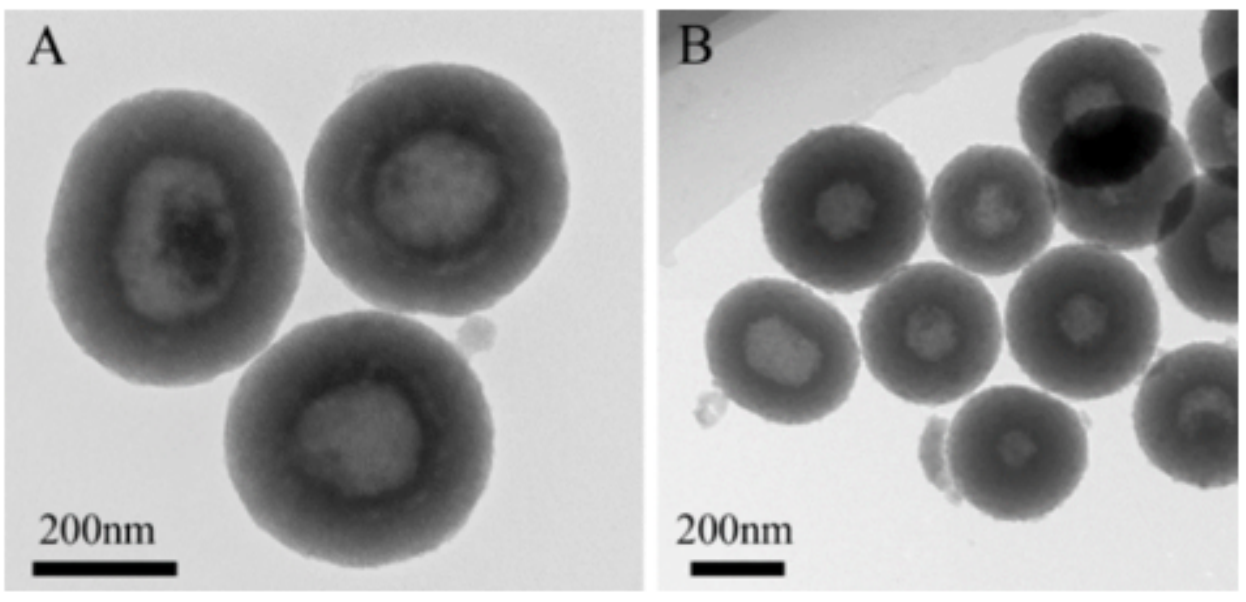

\section{Figure 1}

TEM of (A) HMSS and (B) HMSS-N=N-CS. 

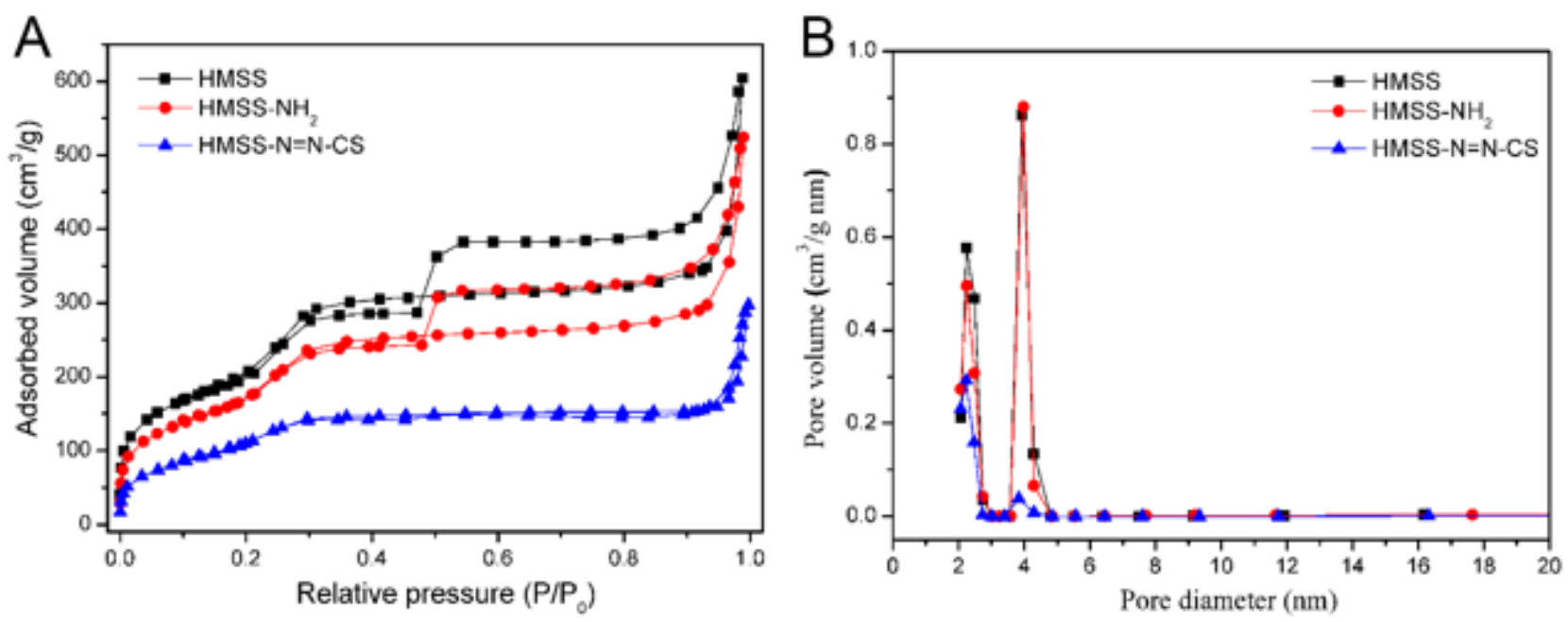

Figure 2

The nitrogen adsorption/desorption isotherms and pore size distributions of HMSS, HMSS-NH2 and HMSS-N=N-CS.

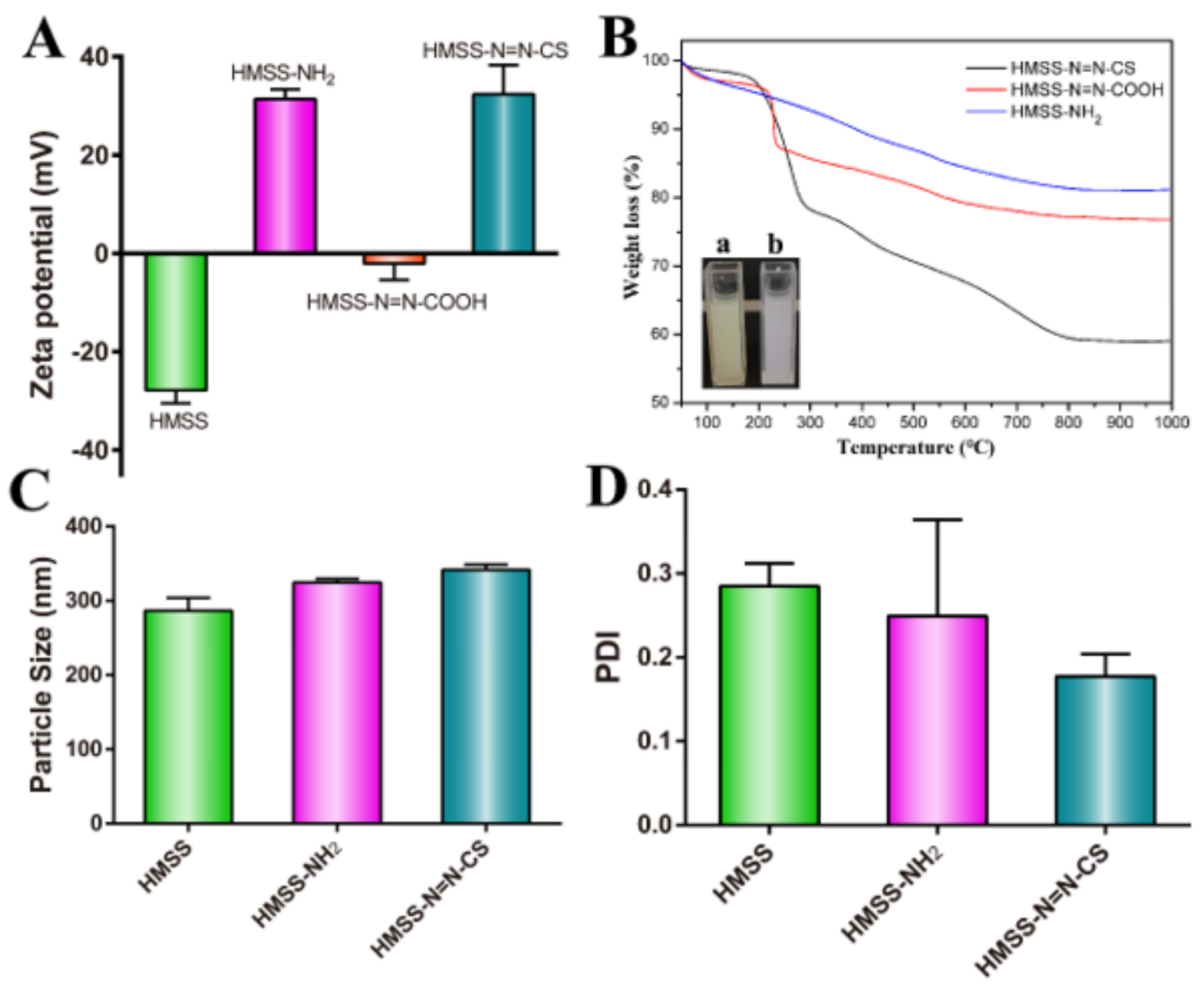

Figure 3 
(A) The corresponding $\xi$ potentials of HMSS, HMSS-NH2, HMSS-N=N-COOH and HMSS-N=N-CS; (B) The TGA curves of HMSS-NH2, HMSS-N=N-COOH and HMSS-N=N-CS (The inset: the photograph of (a) HMSS-N=N-COOH and (b) HMSS-NH2); (C) Size distribution and (D) PDI of HMSS, HMSS-NH2 and HMSS-N=N-CS.

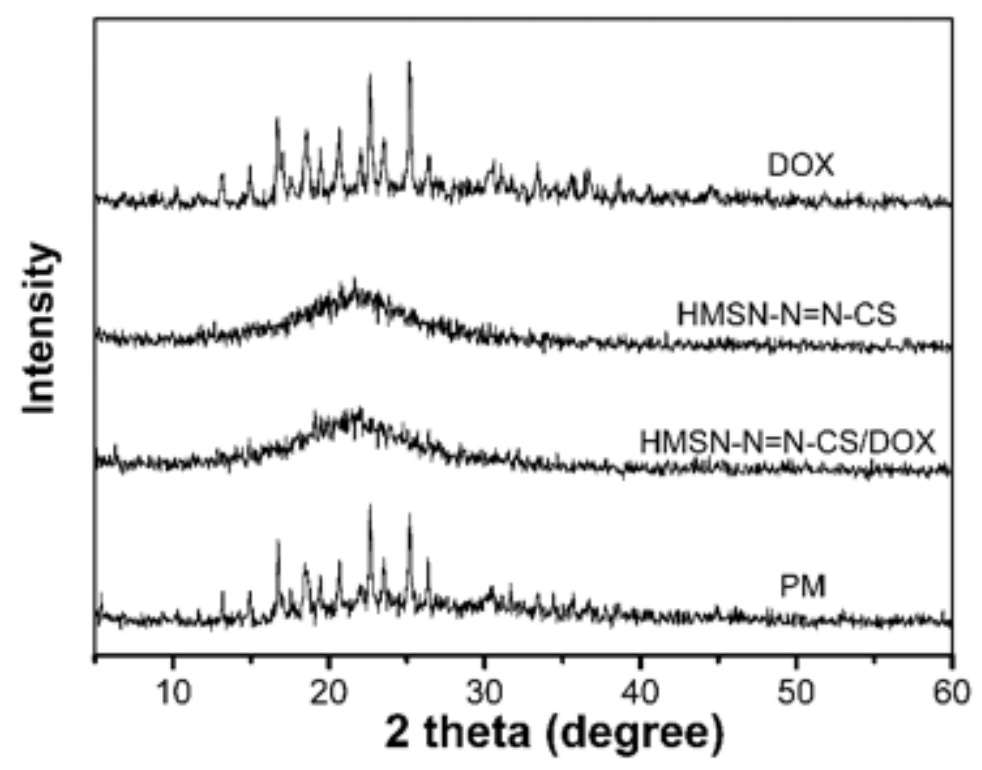

Figure 4

XRD patterns of DOX, HMSS-N=N-CS, HMSS-N=N-CS/DOX and PM.
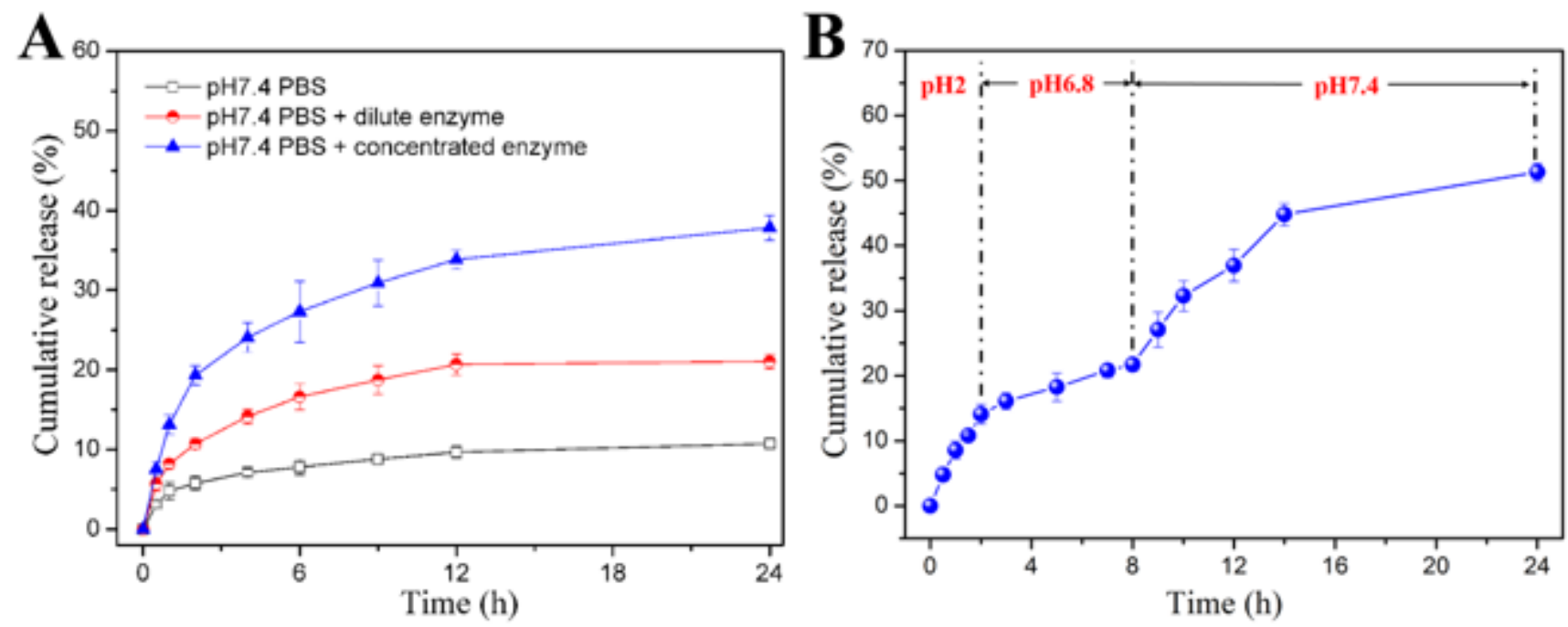

Figure 5

(A) Cumulative release profiles of HMSS-N=N-CS/DOX in pH 7.4 PBS in presence of concentrated and dilute colonic enzyme mixture; and (B) in vitro $\mathrm{pH}$-responsive release behaviors of DOX from HMSS-N=N$\mathrm{CS}$ in the release media of different $\mathrm{pH}$ values. 

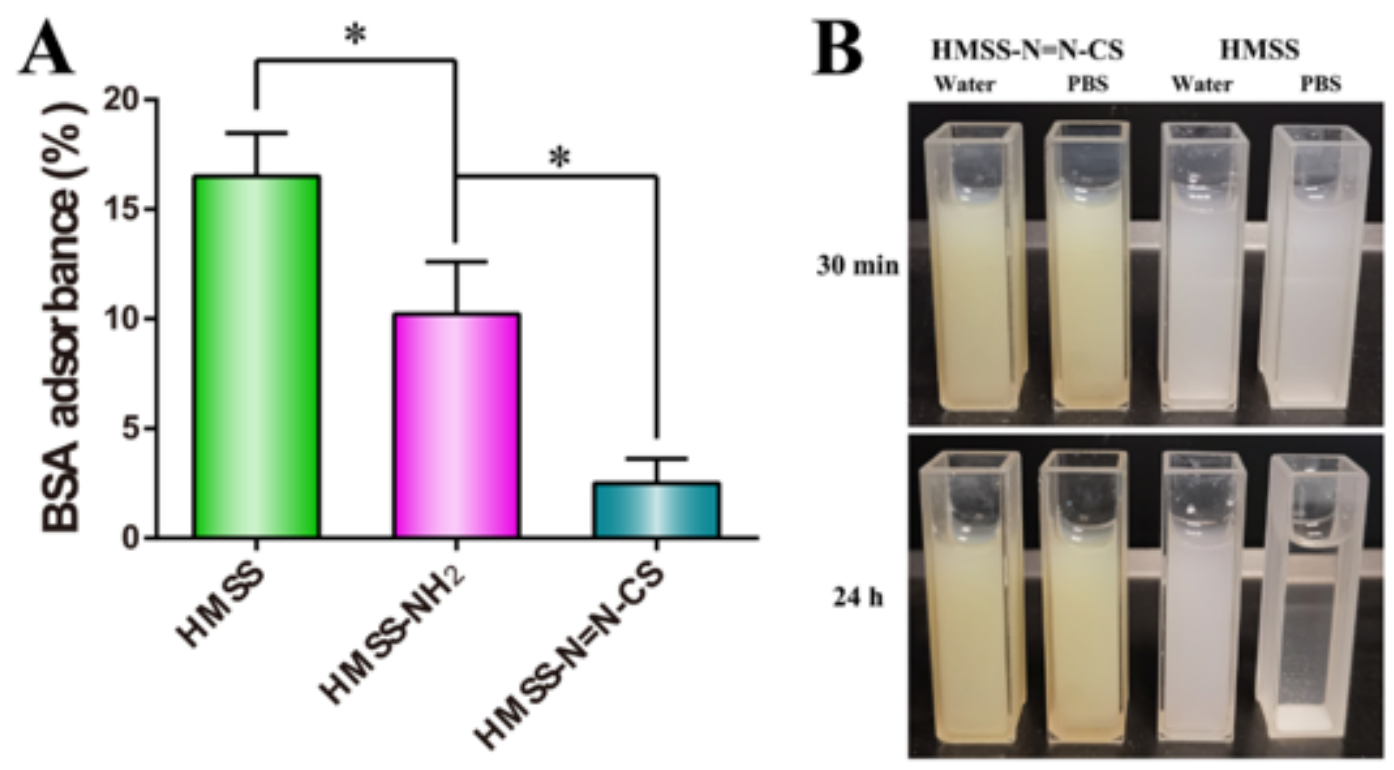

Figure 6

(A) BSA adsorbance amounts of HMSS and HMSS-NH2 and HMSS-N=N-CS. (B) Photograph images of HMSS-N=N-CS and HMSS dispersed in water and PBS with a concentration of $4 \mathrm{mg} / \mathrm{mL}$.

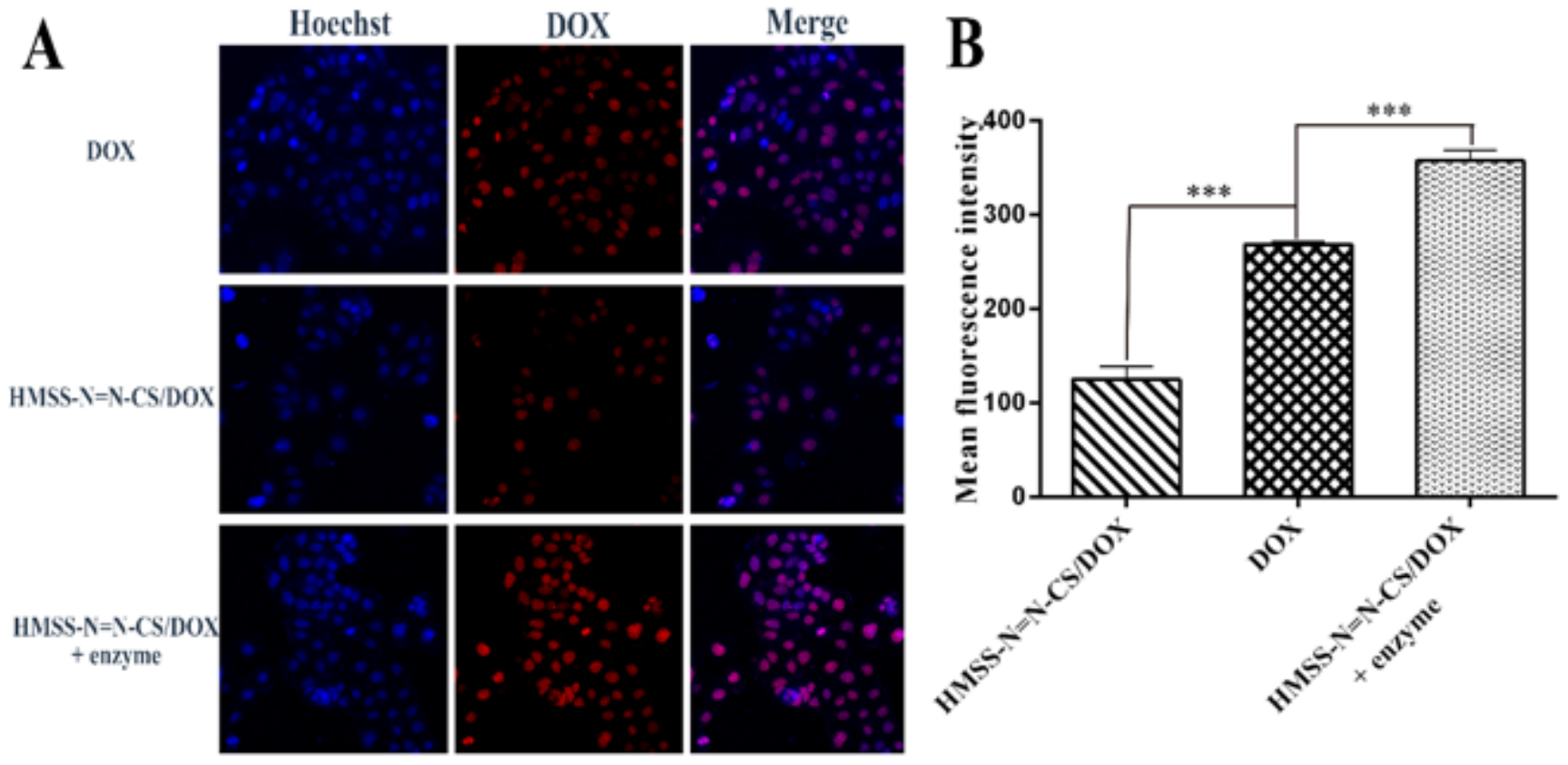

Figure 7

(A) CLSM of Caco-2 cells incubated with different samples. (B) The MFI of DOX, HMSS-N=N-CS/DOX and HMSS-N=N-CS/DOX treated by enzyme measured by FCM in Caco-2 cells. $(n=3, \star \star \star ~ p<0.001)$ 

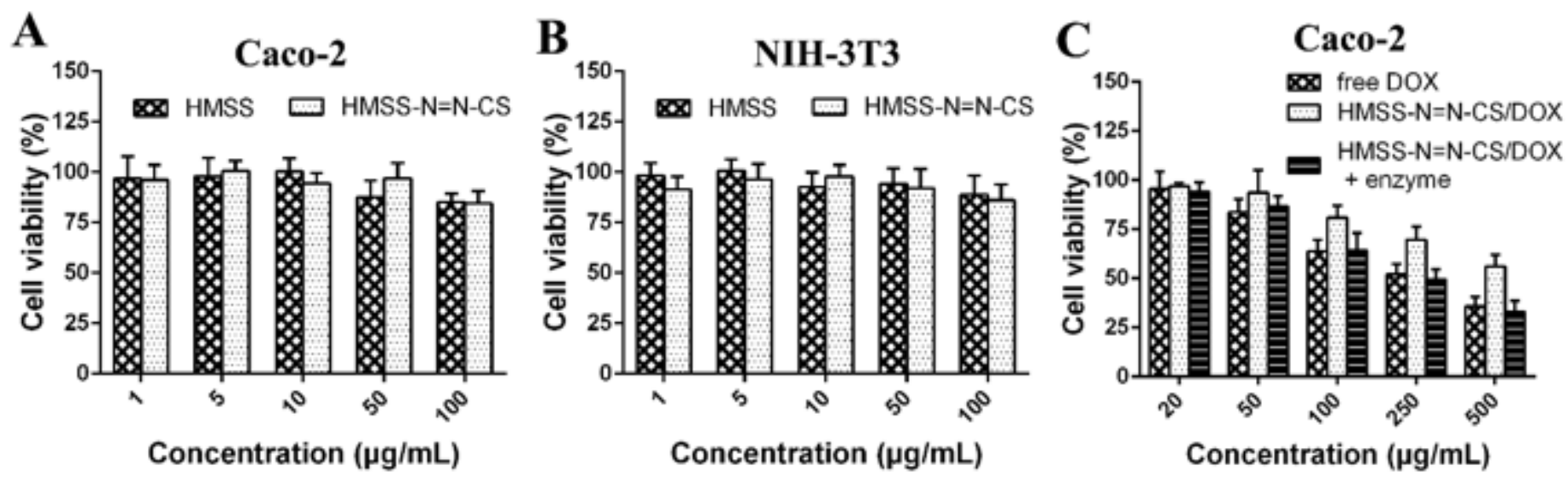

Figure 8

Effect of HMSS and HMSS-N=N-CS on cell proliferation of (A) Caco-2 cells and (B) NIH-3T3 cells for $48 \mathrm{~h}$ by MTT assay; (C) Cytotoxicity of free DOX, HMSS-N=N-CS/DOX and HMSS-N=N-CS/DOX with enzyme against Caco-2 cells with different concentrations for $48 \mathrm{~h}$.
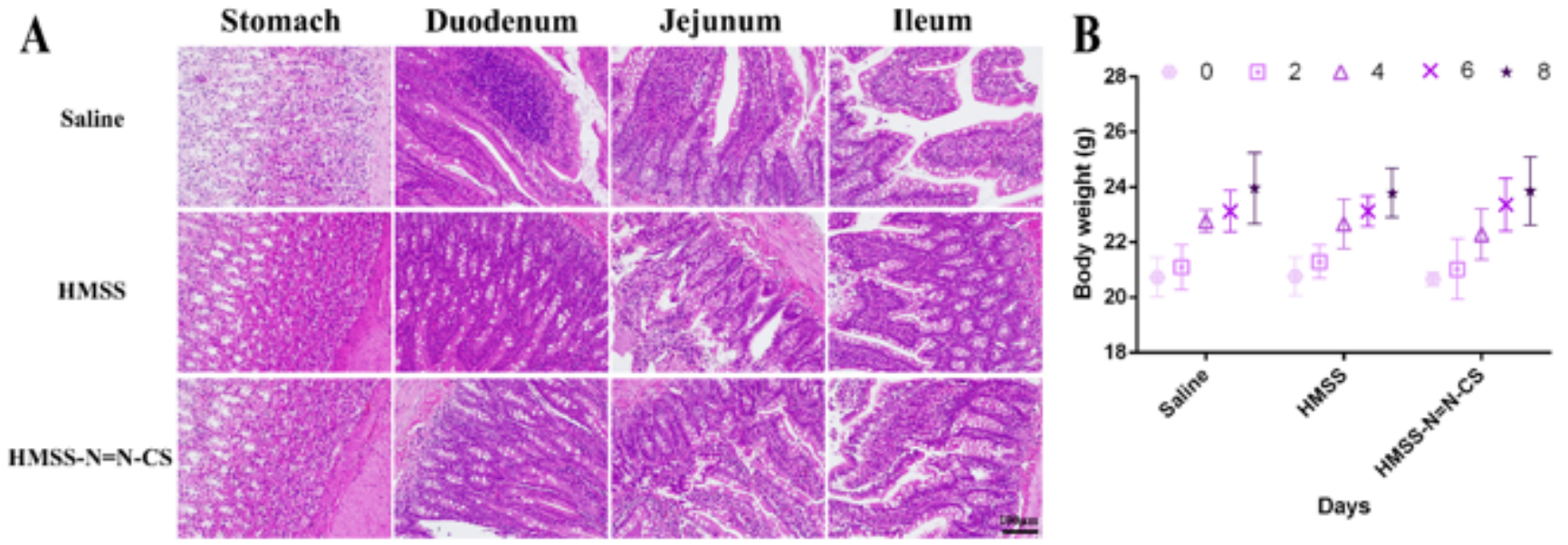

Figure 9

(A) Gastrointestinal mucosa irritation assay after oral administration of HMSS and HMSS-N=N-CS with the dose of $50 \mathrm{mg} / \mathrm{kg}$ for $7 \mathrm{~d}$. (B) The weight changes of mice after oral administration for a week. Data were means $\pm \operatorname{SD}(n=3)$.

\section{Supplementary Files}

This is a list of supplementary files associated with this preprint. Click to download.

- Scheme1.docx 